\title{
Multirate sliding mode disturbance compensation for model predictive control
}

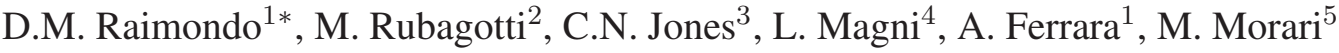 \\ ${ }^{1}$ Dept. of Electrical, Computer and Biomedical Engineering, University of Pavia, 27100 Pavia, Italy \\ ${ }^{2}$ Dept. of Robotics and Mechatronics, Nazarbayev University, 010000 Astana, Kazakhstan \\ ${ }^{3}$ Institute of Mechanical Engineering, Federal Institute of Technology (EPFL), 1015 Lausanne, Switzerland \\ ${ }^{4}$ Civil Engineering and Architecture Department, University of Pavia, 27100 Pavia, Italy \\ ${ }^{5}$ Automatic Control Laboratory, Federal Institute of Technology (ETH), 8092 Zurich, Switzerland
}

\begin{abstract}
SUMMARY
In this paper, a novel hierarchical multirate control scheme for nonlinear discrete-time systems is presented, consisting of a robust Nonlinear Model Predictive Controller (NMPC) and a Multirate Sliding Mode Disturbance Compensator (MSMDC). The proposed MSMDC acts at a faster rate than the NMPC in order to keep the system as close as possible to the nominal trajectory predicted by NMPC despite model uncertainties and external disturbances. The a-priori disturbance compensation turns out to be very useful in order to improve the robustness of the NMPC controller. A dynamic input allocation between MSMDC and NMPC allows to maximize the benefits of the proposed scheme that unites the advantages of sliding mode control (strong reduction of matched disturbances, low computational burden) to those of NMPC (optimality, constraints handling). Sufficient conditions required to guarantee Input-to-State Stability (ISS) and constraints satisfaction by the overall scheme are also provided. Copyright (c) 2013 John Wiley \& Sons, Ltd.

Received ...
\end{abstract}

KEY WORDS: Model Predictive Control; Sliding Mode Control; Uncertain Systems

\section{INTRODUCTION}

Model Predictive Control (MPC) is a control technique able to cope with constraints on control and state variables and to provide an optimal control strategy with respect to a given performance index [32]. In recent years, Nonlinear Model Predictive Control (NMPC) has become an increasingly popular approach for the feedback control of nonlinear systems [10, 20], and a considerable amount of attention has been given to the robustness of the controlled systems with respect to model mismatches and disturbances [16]. Basically, two approaches to tackle this problem exist. The first consists of synthesizing a nominal MPC law (see, e.g., [32]), and checking a posteriori the stability and performance properties of the closed-loop system, in the presence of uncertain terms. The second approach consists of obtaining a-priori guarantees on the stability of the closed-loop system, by explicitly taking into account the information on the disturbance terms. Typically, one can analyze the conditions under which nominal NMPC has inherent robustness properties $[9,23,27,28]$, or synthesize a robust NMPC controller. As for this latter option, the

\footnotetext{
${ }^{*}$ Correspondence to: ${ }^{1}$ Dept. of Electrical, Computer and Biomedical Engineering, University of Pavia, 27100 Pavia, Italy - email \{davide.raimondo@unipv.it\}
} 
main methods are min-max MPC, in which the objective function is minimized for the worst possible realization of the uncertainty while enforcing the satisfaction of constraints for any possible perturbation $[4,14,17,19,31,38]$, or the so-called "tightened constraints NMPC", where robust stability and constraints satisfaction are guaranteed solving the nominal open-loop optimization problem with tightened constraints [13,15,29]. Even though tightened constraints NMPC strongly reduces the computational burden with respect to min-max MPC, its open-loop nature makes it very conservative.

Sliding Mode (SM) control is a well-known robust control technique [39]. Differently from high gain control, which is a continuous control approach often used to solve disturbance rejection problems, SM control, when formulated in the continuous-time framework, is capable of completely rejecting the effect of the so-called "matched disturbances" (i.e. the disturbances that act on the control input channel) with a finite control amplitude [7,39]. The ideal disturbance rejection is attained once the state $x$ belongs to the manifold described by the expression $s(x)=0$, and named "sliding manifold", while the value of $s(x)$ is referred to as "sliding variable". The steering of the state onto the sliding manifold is guaranteed by the SM control law in a finite time interval, and the motion of the state along such a manifold is defined as a "sliding mode". The classical SM approach results in a control law defined on a discontinuous domain, whose application requires the infinitefrequency switching of the control variable. This makes theoretical continuous-time SM not exactly implementable in practice.

The practical implementation of the continuous-time SM control law (that is, its implementation with a non-negligible sampling time) makes the rejection of the matched disturbance not perfect. This happens because the ideal sliding mode enforcement is lost. In this case, the evolution of the state of the closed-loop system occurs within a boundary layer of the selected sliding manifold [39]. Moreover, high-frequency oscillations of the controlled variables can appear, leading to the wellknown chattering effect [40]. As an alternative, a discrete-time design of SM control can be used (see e.g. $[2,37,40]$ and the references therein). This approach aims to compute, at each sampling instant $t$, the value of the control variable capable of ideally steering the state to the sliding manifold in one sampling interval (i.e. $s(t+1)=0$ ). As a consequence, the control law is not defined on a discontinuous domain anymore, thus leading to a reduction of the chattering effect. On the other side, since the control action defined at time $t$ cannot take into account the effect of the unknown disturbance acting at the same time instant, it is impossible to actually steer $s$ to zero in one sampling time. For this reason, as in the case of the practical implementation of the continuous time SM control, discrete-time SM does not guarantee the complete rejection of the matched disturbances. Clearly, a significant reduction of the disturbance can still be achieved provided that the sampling time is short enough.

In recent years, several authors have proposed control strategies that combine sliding mode control and optimal control $[3,8,30,34,41]$. The objective is to come up with a scheme able to provide constraints satisfaction and optimality (typical of MPC) but also good robustness properties with low extra computational burden (typical of SM).

In this paper we present a multi-rate approach where the SM control law, being computationally cheap and more effective with a shorter sampling time, acts at a faster rate than MPC, whose execution rate is limited by the complexity of the constrained optimization problem solved at each sampling time. While an analogous framework has been proposed in [34] for the continuous-time case (with SM control acting at an infinite frequency), here we are interested in providing a control scheme that can be used in practice. Towards this end, we employ a discrete-time sliding mode scheme, called multirate sliding mode disturbance compensator (MSMDC). Similarly to integral sliding mode controllers [1,33,40], the MSMDC controller ensures that the state is kept on a neighborhood of the sliding manifold, thus counteracting matched disturbances, from the initial time instant. However, differently from [34], the discrete-time nature of such approach does not allow for a complete elimination of the matched disturbances. For this reason, even in absence of unmatched disturbances, the combined scheme requires a robust NMPC strategy. In the following, we will consider the tightened constraints NMPC scheme proposed in [13] only. Note that, since the disturbance reduction operated by the SM controller can be determined independently from 
the specific MPC strategy which is employed, the proposed scheme can be applied with the same benefits to any robust MPC control law, as for example min-max MPC. It is well known that minmax MPC can guarantee practical stability only. The practical nature of the stability is a consequence of the worst-case approach of the control action and causes the system to be ultimately bounded even if the real disturbances vanish. The proposed scheme would allow to converge to a smaller region around the equilibrium point by reducing the equivalent disturbance visible to MPC. For a detailed comparison between robust MPC strategies we refer the interested reader to [21,31]. Another robust NMPC scheme that posseses two degrees of freedom is the one proposed in [24]. The approach in [24], presents in the outer loop a controller working in an open-loop fashion, whose action is obtained by solving an optimal control problem off-line (or only when the reference changes). In the inner loop, an MPC controller acts as a disturbance compensator solving on-line a constrained optimizaton. However, the complexity of the optimization does not allow for high sampling rates. Indeed, the MSMDC we propose can be added to [24] as a further layer, thus improving the disturbance rejection even further. Note that the interest in multirate control schemes has increased in recent years (see, e.g., [12]), in particular in the sliding mode community (see, e.g., [11]).

Another problem that can arise in practice (and that was not taken into account in [34]) when implementing sliding mode control, is that input bounds can limit the effectiveness of $\mathrm{SM}$ in counteracting the disturbances. In the following we compute bounds on the equivalent disturbance visible by the MPC as a function of the maximal input allocated to the SM controller. A further contribution of the hierarchical scheme here presented, for which the estimation of these disturbance bounds is essential, is a dynamic input allocation between SM and MPC. Under suitable assumptions, tightened constraints NMPC guarantees Input-to-State Stability and constraints satisfaction provided that the disturbance is small enough. MSMDC allows to reduce the equivalent disturbance visible to NMPC by acting at a faster rate, thus resulting beneficial in cases when the amplitude of the disturbance is too large. As a counter effect, static input allocation could lead to a reduction of the domain of attraction, in which recursive feasibility for the MPC controller is a-priori guaranteed. A dynamic allocation allows the adaptation of input partitioning according to the state conditions. When the state is far from the origin, MSMDC is provided with the minimum actuation range that guarantees MPC feasibility. On the other side, when the state is closer to the origin and MPC requires less input, more input is allocated to the MSMDC in order to provide a stronger disturbance rejection.

The paper is organized as follows: Section 2 introduces the main notations used through the paper, while Section 3 introduces the considered class of systems and the formulation of the problem in question. Sections 4 and 4.3 describe the MSMDC design while section 5 the MPC control law. Finally, a simulation example is shown in Section 6 and some conclusions are drawn in Section 7.

This article extends the preliminary results in [35] by providing a more general theoretical development, an improved computation of the disturbance bounds and a dynamic input allocation between MPC and MSMDC control.

\section{NOTATION}

Let $\mathbb{R}, \mathbb{R}_{\geq 0}, \mathbb{Z}$ and $\mathbb{Z}_{\geq 0}$ denote the sets of real, non-negative real, integer and non-negative integer numbers, respectively. Given a matrix $B$, its element on the $i$-th row and $j$-th column is denoted by $B_{i, j}$. The set of sequences of $w$, whose values belong to a compact set $\mathcal{W}$, is denoted by $\mathcal{M}_{\mathcal{W}}$. Given a signal $w$, the signal sequence is denoted by $\mathbf{w} \triangleq\left\{w_{0}, w_{1}, \cdots\right\}$, where the cardinality of the sequence is inferred from the context. The absolute value and the Euclidean norm are denoted as $|\cdot|$ and $\|\cdot\|$, respectively, while $\|\mathbf{w}\|_{\mathcal{L}_{\infty}} \triangleq \sup _{k \geq 0}\left\{\left\|w_{k}\right\|\right\}$. Given $a \in \mathbb{R}_{\geq 0}$, the Euclidean norm ball in $\mathbb{R}^{n}$ is defined as $\mathcal{B}_{a} \triangleq\left\{x \in \mathbb{R}^{n}:\|x\| \leq a\right\}$. Given two sets $A \subseteq \mathbb{R}^{n}$ and $B \subseteq \mathbb{R}^{n}$, the Pontryagin difference set $C$ is defined as $C=A \sim B \triangleq\left\{x \in \mathbb{R}^{n}: x+\xi \in \bar{A}, \forall \xi \in B\right\}$. Given a function $\psi(\cdot)$, its $r$-th iterate is indicated as $\psi^{\circ r}(\cdot) \triangleq \psi \circ \psi \circ \cdots \circ \psi(\cdot)$, while $\psi^{(j)}(\cdot)$ denotes its $j$ - th component and $\psi^{[i, j]}(\cdot)$ the components from $i$ to $j$. A function $\gamma: \mathbb{R}_{\geq 0} \rightarrow \mathbb{R}_{\geq 0}$ is a " $\mathcal{K}$-function" if it is 
continuous, positive definite and strictly increasing. A function $\gamma: \mathbb{R}_{\geq 0} \rightarrow \mathbb{R}_{\geq 0}$ is a $\mathcal{K}_{\infty}$-function if it is a $\mathcal{K}$-function and $\gamma(s) \rightarrow+\infty$ as $s \rightarrow+\infty$. A function $\beta: \mathbb{R}_{\geq_{0}} \times \mathbb{Z}_{\geq_{0}} \rightarrow \mathbb{R}_{\geq_{0}}$ is a $\mathcal{K} \mathcal{L}$-function if, for each fixed $t \geq 0, \beta(\cdot, t)$ is of class $\mathcal{K}$, for each fixed $s \geq 0, \beta(s, \cdot)$ is decreasing and $\beta(s, t) \rightarrow 0$ as $t \rightarrow \infty$.

\section{PROBLEM FORMULATION}

\subsection{Description of the system}

Consider a discrete-time nonlinear system

$$
x_{t+1}=f\left(x_{t}\right)+B u_{t}+d_{t}
$$

where $t \in \mathbb{Z}_{\geq 0}, x \in \mathbb{R}^{n}$ is the state vector, $u \in \mathbb{R}^{m}$ is the control vector, $f: \mathbb{R}^{n} \rightarrow \mathbb{R}^{n}$ is a nonlinear function, $B \in \mathbb{R}^{n \times m}$ is a constant matrix with full column-rank (we assume $m \leq n$ ), and $d \in \mathbb{R}^{n}$ represents the external disturbance.

\section{Assumption 1}

The following holds for system (1):

1. matrix $B$ is in the form

$$
B=\left[\begin{array}{l}
\mathbf{I} \\
\mathbf{0}
\end{array}\right],
$$

where $\mathbf{I} \in \mathbb{R}^{m \times m}$ is an identity matrix, and $\mathbf{0} \in \mathbb{R}^{n-m \times m}$ is a zero matrix;

2. $f(0)=0$;

3. each component of the control variable $u^{(j)}$ is bounded as $\left|u^{(j)}\right| \leq U^{(j)}, j=1, \ldots, m$, where $U^{(j)}$ are positive constants;

4. each component $d^{(j)}$ of the disturbance $d$ admits a bound $\left|d^{(j)}\right| \leq \delta^{(j)}, j=1, \cdots, n$.

The euclidean norm of the maximum disturbance vector can be defined as

$$
\gamma_{\delta}=\|\delta\|, \quad \delta \triangleq\left[\begin{array}{llll}
\delta^{(1)} & \delta^{(2)} & \ldots & \delta^{(n)}
\end{array}\right]^{\top} .
$$

Our objective is to design a control law which steers asymptotically $x_{t}$ to a set including the origin despite the presence of the disturbance term $d_{t}$, and considering that we want to enforce the state constraint

$$
x_{t} \in X \subseteq \mathbb{R}^{n}
$$

for all $t \in \mathbb{Z}_{\geq 0}$, where $X$ is a compact set including the origin in its interior. The following continuity hypothesis on system (1) is introduced:

Assumption 2

Each component $j=1, . ., n$ of function $f(\cdot)$, namely $f^{(j)}(\cdot)$, satisfies the following continuity condition

$$
\left|f^{(j)}(a)-f^{(j)}(b)\right| \leq \eta_{(j)}(\|a-b\|), \forall a, b \in X
$$

where $\eta_{(j)}(\cdot)$ is a $\mathcal{K}$-function.

Note that this requirement is weaker than the widely used Lipschitz condition, and obtainable as a particular case with $\eta_{(j)}(s)=L_{(j)} \cdot s, L_{(j)}$ being a positive constant. Assumption 2 also implies that there exists a $\mathcal{K}$-function $\eta$ s.t.

$$
\|f(a)-f(b)\| \leq \eta(\|a-b\|), \forall a, b \in X
$$

given, for instance, by $\eta(\cdot)=\sqrt{\sum_{j=1}^{n} \eta_{(j)}^{2}(\cdot)}$. For the same reason, there exists a $\mathcal{K}$-function $\eta^{[m+1, n]}$ such that, denoting with $f^{[m+1, n]} \triangleq\left[\begin{array}{llll}f^{(m+1)} & f^{(m+2)} & \ldots & f^{(n)}\end{array}\right]^{\top}$, the following 
holds

$$
\left\|f^{[m+1, n]}(a)-f^{[m+1, n]}(b)\right\| \leq \eta^{[m+1, n]}(\|a-b\|), \forall a, b \in X .
$$

Finally, the nominal evolution of system (1) at time $t+1$ calculated with respect to an initial state $x_{t_{0}}$ and an input sequence $u_{t_{0}}, u_{1}, \ldots, u_{t-1}$, with $t_{0}<t$, is denoted with $z_{t+1 \mid t_{0}}$ and expressed as

$$
z_{t+1 \mid t_{0}}=f\left(z_{t \mid t_{0}}\right)+B u_{t},
$$

where $z \in \mathbb{R}^{n}$, and $z_{t_{0} \mid t_{0}} \triangleq x_{t_{0}}$.

\subsection{Considerations on the normal form}

In the regular form determined by the structure of matrix $B$, the separation between the so-called "matched" and "unmatched" disturbances [7] is apparent. The first $m$ components of $d$ form the matched disturbance vector, i.e. the vector of the disturbances acting on the input channel, while the remaining components form the unmatched vector. Note that the requirement of regular form representation does not constitute a strong limitation. Since matrix $B$ is constant and full columnrank, it is possible to find a suitable linear state transformation in order to transform a generic system with constant input matrix into the regular form representation. Note that state and input constraints will change according to the applied transformation.

\subsection{Overview of the control strategy}

The proposed control strategy consists of two components which cooperate so as to attain the control objective (see Figure 1). Thus, the control variable $u$ can be split into two parts, as

$$
u_{t}=\underline{u}_{t}+\bar{u}_{t},
$$

where $\underline{u}_{t}$ and $\bar{u}_{t}$ are respectively generated by the HLC and the MSMDC. The HLC acts at a slower rate than the MSMDC: it samples the state value each $M$ steps (where $M$ is a positive constant) and generates a control variable that is kept constant over this time interval, i.e. $\underline{u}_{M(t+1)-i}=\underline{u}_{t M}, i=$ $1, \ldots, M$. Due to the bounds on the control variables (see Assumption 1), we allocate a maximum control amplitude to $\underline{u}_{t}$ and $\bar{u}_{t}$, as follows.

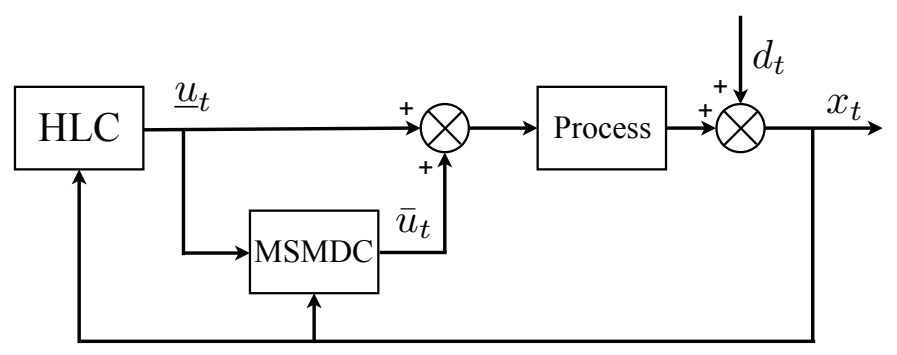

Figure 1. Scheme of the overall hierarchical control system.

\section{Assumption 3}

Each component of $\underline{u}_{t}^{(j)}$ and $\bar{u}_{t}^{(j)}$ is bounded by $\underline{U}^{(j)}$ and $\bar{U}^{(j)}$ respectively, $j=1, \ldots, m$, where $\underline{U}^{(j)}$ and $\bar{U}^{(j)}$ are design parameters such that $\underline{U}^{(j)}+\bar{U}^{(j)} \leq U^{(j)}$.

\subsection{Representation of the "M-sampled" system}

Now we define a representation of the system for the HLC (i.e. the " $M$-sampled" system), using the new discrete-time variable $k \in \mathbb{Z}_{\geq 0}$, as

$$
\xi_{k+1}=\phi\left(\xi_{k}, \mu_{k}\right)+w_{k},
$$


where $\xi_{k} \triangleq x_{k M}$ and $\mu_{k} \triangleq \underline{u}_{k M}$. Note that the action of $\bar{u}$ is not explicitly considered in the $M$ sampled system. Indeed, the MSMDC action is "transparent" to the HLC. Its action is considered merged with that of the disturbances, determining an "equivalent" disturbance, which is the actual disturbance the HLC has to cope with. The function $\phi(\cdot, \cdot)$ must be found according to the information available on the nominal evolution of the system in (8), while $w_{k} \in \mathbb{R}^{n}$ (which represents the difference between the nominal and perturbed evolutions) must be found according to the information available on system (1). Note that the change in the name of the variables to account for a change in the sampling time is a standard procedure in multirate systems, as can be found in [6].

To find an expression for $\phi(\cdot, \cdot)$, consider the evolution of the nominal system (8) each $M$ steps. To this purpose, after defining $g_{\underline{u}_{k M}}\left(x_{k M}\right) \triangleq f\left(x_{k M}\right)+B \underline{u}_{k M}$, one has only to iterate this function $M$ times to obtain the nominal evolution of the system at the next sampling time of the HLC, as $z_{M(k+1) \mid k M}=g_{\underline{u}_{k M}}^{\circ M}\left(z_{k M \mid k M}\right)$, keeping in mind that $z_{k M \mid k M}=x_{k M}$. Noting that the resulting system is not control-affine anymore, $\phi(\cdot, \cdot)$ is obtained as

$$
\phi\left(\xi_{k}, \mu_{k}\right) \triangleq g_{\underline{u}_{k M}}^{\circ M}\left(x_{k M}\right) .
$$

\section{Lemma 1}

Consider system (1) with $\bar{u}_{t}=0, \forall t \in \mathbb{Z}_{\geq 0}$, and $M \geq 1$. An upper bound on the disturbance term in (10) is given by

$$
\left|w^{(j)}\right| \leq \sigma_{M}^{(j)}
$$

where

$$
\begin{gathered}
\sigma_{i}^{(j)} \triangleq\left\{\begin{array}{ll}
0 & \text { if } i=0 \\
\eta_{(j)}\left(\varrho_{i-1}\right)+\delta^{(j)} & \text { if } i>0
\end{array}, \quad \zeta_{i} \triangleq\left\{\begin{array}{ll}
0 & \text { if } i=0 \\
\eta\left(\varrho_{i-1}\right)+\gamma_{\delta} & \text { if } i>0
\end{array},\right.\right. \\
\varrho_{i} \triangleq \min \left(\left\|\sigma_{i}\right\|, \zeta_{i}\right), \quad \sigma_{i} \triangleq\left[\begin{array}{llll}
\sigma_{i}^{(1)} & \sigma_{i}^{(2)} & \ldots & \sigma_{i}^{(n)}
\end{array}\right]^{\top}, \\
i=1, \cdots, M \text {, and } j=1, \cdots, n \text {, with } \eta_{(j)} \text { and } \eta \text { as defined in (5), (6). }
\end{gathered}
$$

Proof: See the appendix.

Henceforth, we will refer to the euclidean norm of the maximum disturbance vector $w$ as $\gamma_{w}$.

Lemma 2

If Assumption 2 holds, there exists a $\mathcal{K}$-function $\lambda(\cdot)$ such that function $\phi(\cdot, \cdot)$ defined in (11) satisfies the following continuity condition

$$
\|\phi(a, \mu)-\phi(b, \mu)\| \leq \lambda(\|a-b\|), \forall a, b \in X, \mu \in U
$$

Proof: The result is easily obtained iterating (8) $M$ times using (6), with $\lambda(\cdot) \triangleq \eta^{\circ M}(\cdot)$.

\section{THE SLIDING MODE DISTURBANCE COMPENSATOR}

\subsection{General formulation}

The problem dealt with in this section is to design the control component $\bar{u}_{t}$ in (9) so as to obtain an effective disturbance reduction. The concept of sliding mode control in discrete time is not as established as it is in continuous time, and different approaches have been described in the literature. A possibility to obtain a discrete-time sliding mode controller is to directly discretize a continuoustime controller. This would lead to the definition of a discontinuous control law, which would introduce chattering into the closed-loop system. To avoid such a problem, we employ here the idea of discrete-time sliding mode for which the reader is referred to [40, Chap. 9]. The idea consists of defining $\bar{u}_{t}$ in order to steer to zero a suitably-defined sliding manifold in one time instant: we apply this concept here by defining a sliding manifold which accounts for the difference between the predicted and actual evolutions of the system. More precisely, with respect to system (1), we 
define the sliding manifold

$$
s_{t M+i}=C\left(x_{t M+i}-z_{t M+i \mid t M}\right), i=1, \cdots, M
$$

where

$$
C=\left[\begin{array}{ll}
\mathbf{I} & \mathbf{0}
\end{array}\right]
$$

where $\mathbf{I} \in \mathbb{R}^{m \times m}$ is an identity matrix, while $\mathbf{0} \in \mathbb{R}^{m \times n-m}$ is a zero matrix. Of course, different choices could be made to determine $C$. In the remainder of the paper we will show that this particular choice guarantees an overall disturbance reduction if basic assumptions are satisfied. With this choice of matrix $C$, the action of $\bar{u}_{t}$ is aimed at steering to zero in one step the difference between the ideal and perturbed evolutions of the states on which the matched disturbance is acting. Regardless of the choice of $C$, the value of $s$ one step ahead is given by

$$
\begin{aligned}
s_{t M+i+1}=C\left(x_{t M+i+1}-z_{t M+i+1 \mid t M}\right) & \\
=C\left(f\left(x_{t M+i}\right)+B\left(\underline{u}_{t M+i}+\right.\right. & \left.\left.\bar{u}_{t M+i}\right)+d_{t M+i}-f\left(z_{t M+i \mid t M}\right)-B \underline{u}_{t M+i}\right) \\
& \left.=C\left(f\left(x_{t M+i}\right)-f\left(z_{t M+i \mid t M}+B \bar{u}_{t M+i}\right)+d_{t M+i}\right)\right) .
\end{aligned}
$$

Notice that the nominal evolution of the state (i.e., $z_{t}$ ) does not consider the presence of the MSMDC action $\bar{u}_{t}$, which is instead present in the evolution of the actual state $x_{t}$. In order to define $\bar{u}_{t M+i}$ that will steer $s_{t M+i+1}$ to zero, according to the definition of discrete-time sliding mode control in [40, Chap. 9], we need to define an estimate $\hat{d}_{t M+i}$ of the disturbance (since the value of $d_{t M+i}$ is unknown). We assume now that the value of $\hat{d}_{t M+i}$ is known (suitable methods to obtain it will be described in Section 4.3), therefore obtaining

$$
\bar{u}_{t M+i}=-(C B)^{-1} C\left(f\left(x_{t M+i}\right)-f\left(z_{t M+i \mid t M}\right)+\hat{d}_{t M+i}\right) .
$$

Substituting the expressions of $B$ in (2) and $C$ in (14) into (15), and expressing the value of $\bar{u}_{t}$ component-wise, the control action acting on the $j-t h$ state component is

$$
\bar{u}_{t M+i}^{(j)}=-f^{(j)}\left(x_{t M+i}\right)+f^{(j)}\left(z_{t M+i \mid t M}\right)-\hat{d}_{t M+i}^{(j)}
$$

at any time instant and for all $j=1, \cdots, m$. The matched disturbance at each time instant $t M+i$ is then changed from $d_{t M+i}^{(j)}$ in the uncompensated case, to $d_{t M+i}^{(j)}+\bar{u}_{t M+i}^{(j)}$ in the compensated case, this latter being defined in (16).

\subsection{Determination of the equivalent disturbance bounds}

In the following, we provide a bound on the equivalent disturbance acting on the $M$-sampled system as a function of the bound on the MSMDC control amplitude. First, we define the maximum error for each state component as

$$
e^{(j)} \triangleq \max _{t}\left|d_{t}^{(j)}-\hat{d}_{t}^{(j)}\right|, j=1, \ldots, n
$$

and introduce the following assumption.

Assumption 4

An upper bound on the estimation error is given for each matched component, as

$$
e^{(j)} \leq \delta^{(j)}, \quad\left|\hat{d}_{t}^{(j)}\right| \leq \delta^{(j)},
$$

for all $t \in \mathbb{Z}_{\geq 0}$, and all $j=1, \ldots, m$.

Before stating the main result of the paper, we formulate the following intermediate lemma that provides a bound on the input amplitude required by the MSMDC, in order to maximally compensate for the matched disturbances. 


\section{Lemma 3}

For system (1), applying the MSMDC control law (16), an upper bound on the disturbance term in (10) can be computed as

$$
\left|w^{(j)}\right| \leq \hat{\sigma}_{M}^{(j)}
$$

where

$$
\begin{aligned}
& \hat{\sigma}_{i}^{(j)} \triangleq\left\{\begin{array}{llr}
0 & \text { if } i=0 \\
e^{(j)} & j=1, . ., m & \text { if } i>0 \\
\eta_{(j)}\left(\varrho_{i-1}\right)+\delta^{(j)} & j=m+1, . ., n
\end{array}\right. \\
& \hat{\varrho}_{i} \triangleq \min \left(\left\|\hat{\sigma}_{i}\right\|, \hat{\zeta}_{i}\right), \quad \hat{\zeta}_{i} \triangleq\left\{\begin{array}{ll}
0 & \text { if } i=0 \\
\eta^{[m+1, n]}\left(\hat{\varrho}_{i-1}\right)+\gamma_{e} & \text { if } i>0
\end{array},\right.
\end{aligned}
$$

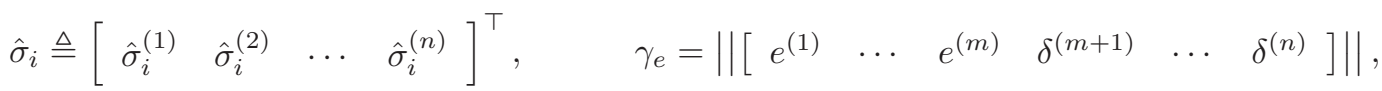

$$
\begin{aligned}
& i=1, \cdots, M, j=1, \cdots, n \text {, with } \eta_{(j)} \text { and } \eta^{[m+1, n]} \text { as defined in (5), (7). }
\end{aligned}
$$

Also, the maximum amplitude required to each component of the MSMDC control variable $\bar{u}^{(j)}$ is equal to

$$
\bar{U}_{R e q(M-1)}^{(j)}=\sum_{q=1}^{m} \eta_{(q)}\left(\hat{\varrho}_{M-1}\right)+\delta^{(q)} .
$$

Proof: See the appendix.

Now, the preliminary result in Lemma 3 is used to obtain the main result of the paper: the computation of a bound on the equivalent disturbance acting on the $M$-sampled system when an a-priori bound is given on the MSMDC control action.

\section{Theorem 1}

For system (1), applying the MSMDC control law (16), the bound on the control input required to the MSMDC in order to compensate for the matched disturbance in the interval $t M, \ldots, t M+i+1$ is equal to

$$
\bar{U}_{\operatorname{Req}(i)}^{(j)}=\eta_{(j)}\left(\hat{\varrho}_{i}\right)+\delta^{(j)} .
$$

Define $\mathcal{I}=\left\{i \in\{0, \ldots, M-1\}\right.$ such that $\bar{U}_{R e q(i)}^{(j)} \geq \bar{U}^{(j)}$, for some $\left.j \in\{1, \ldots, m\}\right\}$. If $\mathcal{I} \neq \emptyset$, define $\underline{i}=\min _{i \in \mathcal{I}} i$. Then, an upper bound on the disturbance term in (10) can be computed as

$$
\left|w^{(j)}\right| \leq \breve{\sigma}_{M}^{(j)}
$$

where

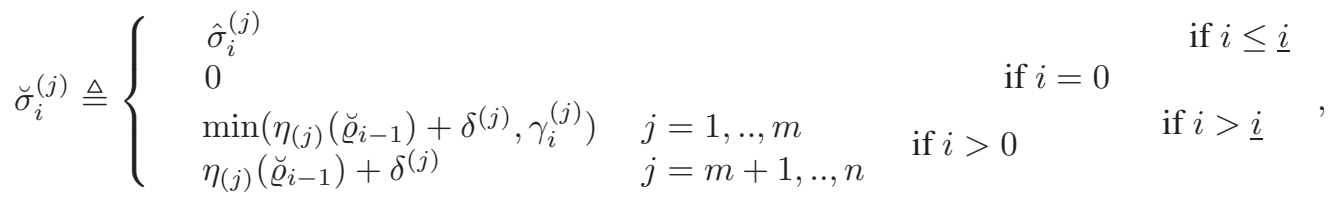

$$
\begin{aligned}
& \breve{\varrho}_{i} \triangleq\left\{\begin{array}{ll}
\hat{\varrho}_{i} & \text { if } i \leq \underline{i} \\
\min \left(\| \breve{\sigma}_{i}|| \breve{\zeta}_{i}\right) & \text { if } i>\underline{i}
\end{array} \quad \gamma_{i}^{(j)} \triangleq \max \left(e^{(j)}, \eta_{(j)}\left(\breve{\varrho}_{i-1}\right)+\delta^{(j)}-\left|\bar{B}_{j, j}\right| \bar{U}^{(j)}+e^{(j)}\right),\right. \\
& \breve{\zeta}_{i} \triangleq\left\{\begin{array}{ll}
\hat{\zeta}_{i} & \text { if } i \leq \underline{i} \\
0 & \text { if } i=0 \\
\eta\left(\breve{\varrho}_{i-1}\right)+\gamma_{0} & \text { if } i>0
\end{array} \quad \text { if } i>\underline{i} \quad, \quad \breve{\sigma}_{i} \triangleq\left[\begin{array}{llll}
\breve{\sigma}_{i}^{(1)} & \breve{\sigma}_{i}^{(2)} & \ldots & \breve{\sigma}_{i}^{(n)}
\end{array}\right]^{\top},\right.
\end{aligned}
$$




$$
\begin{aligned}
& \gamma_{0}=\left\|\left[\begin{array}{cccccc}
\delta_{r e d}^{(1)} & \cdots & \delta_{\text {red }}^{(m)} & \delta^{(m+1)} & \cdots & \delta^{(n)}
\end{array}\right]\right\|, \quad \begin{array}{l}
\delta_{r e d}^{(j)} \triangleq \min \left(\delta^{(j)}, \breve{\delta}^{(j)}\right) \\
\delta^{(j)} \triangleq \max \left(e^{(j)}, \delta^{(j)}-\left|\bar{B}_{j, j}\right| \bar{U}^{(j)}+e^{(j)}\right)
\end{array} \\
& i=1, \cdots, M, j=1, \cdots, n \text {, with } \eta_{(j)} \text { as defined in (5). }
\end{aligned}
$$

Proof: See the appendix.

In the remaining of the paper, the euclidean norm of the maximum disturbance vector $w$ visible by the high-level controller will be denoted as

$$
\gamma_{w}=\left\|\breve{\sigma}_{M}\right\|, \quad \breve{\sigma}_{M} \triangleq\left[\begin{array}{cccc}
\breve{\sigma}_{M}^{(1)} & \breve{\sigma}_{M}^{(2)} & \cdots & \breve{\sigma}_{M}^{(n)}
\end{array}\right]^{\top}
$$

while the set of all possible equivalent disturbances as $\mathcal{W}=\left\{w \in \mathbb{R}^{n} \mid w^{(i)} \leq \breve{\sigma}_{M}^{(i)}, i=1, \ldots, n\right\}$.

Remark 1

It is straightforward to see that the bound in equation (20) is smaller than the bound (12) obtained in Lemma 1 and larger than the bound (17) in Lemma 3.

\section{Remark 2}

The choice of the ratio between the MSMDC and the NMPC sampling times (the variable $M$ ) affects the bound on the equivalent disturbance visible by the NMPC as well as the bound on the MSMDC input required for the compensation. Note that, since the computation of functions $\eta$ is rather conservative in general, a reduction of the MSMDC sampling time does not necessarily guarantee an improvement of the control quality in terms of robust stability, performance and domain of attraction.

\section{Remark 3}

As for the convergence of the system to the sliding manifold, by definition of matrix $C$, one has $\left\|s_{t}\right\| \leq \|\left[\begin{array}{lll}e^{(1)} & \cdots & e^{(m)}\end{array} \|\right.$, for all $t \in \mathbb{Z}_{\geq 0}$. Indeed, as expected for any discrete-time sliding mode approach, the system state is kept on a boundary layer of the sliding manifold, which means that a quasi-sliding mode [26] is enforced.

\subsection{Disturbance estimation}

The simplest solution for disturbance estimation is to assume that $\hat{d}_{t}=0$. In this case, Assumption 4 is satisfied with $e^{(j)}=\delta^{(j)}$, (and, trivially, $0 \leq \delta^{(j)}$ ), for all $j=1, \ldots, m$. Another possible estimate is obtained using the disturbance value at the previous time instant $d_{t-1}^{(j)}=x_{t}^{(j)}-f^{(j)}\left(x_{t-1}\right)-$ $u_{t-1}^{(j)}, j=1, \ldots, m$, and then defining $\hat{d}_{t}^{(j)}=d_{t-1}^{(j)}$. This is a typical choice in discrete-time sliding mode control (see, e.g. [1]), since it is reasonable to assume that the process disturbances are slowly varying: the resulting disturbance upper bound depends on the possible variation of the disturbance term in one time instant. In order to satisfy Assumption 4 in this case, an upper bound on the variation of the each matched component is needed, as $\Delta d^{(j)} \triangleq \max _{t}\left|d_{t}^{(j)}-d_{t-1}^{(j)}\right| \leq \delta^{(j)}$, $j=1, \cdots, m$. This leads to the satisfaction of Assumption 4, with $e^{(j)}=\Delta d^{(j)}$, and $\left|\hat{d}_{t}^{(j)}\right|=\delta^{(j)}$, for all $j=1, \ldots, M$. Note that, the disturbance reduction with respect to the case without MSMDC is guaranteed if $\Delta d^{(j)} \leq \delta^{(j)}$, for all $j=1, \ldots, m$. Other strategies can be applied, depending on the information available on the system, as far as Assumption 4 is satisfied.

\section{THE ROBUST OPEN-LOOP NMPC STRATEGY}

Robust NMPC is a widely used technique in order to ensure robust constraint satisfaction and recursive feasibility in spite of uncertainties and disturbances (see e.g. [16]). In this section, the tightened constraints MPC scheme proposed in [13] is exploited for the HLC of system (10). 


\subsection{Summary of robust MPC strategy}

Given the state constraints $X$, the bound $\gamma_{w}$ on the Euclidean norm of the disturbance term, and the time step $k \geq 0$, define the tightened constraints as follows

$$
\begin{aligned}
& B_{\gamma_{w}}^{\nu-k} \triangleq\left\{z \in \mathbb{R}^{n}:\|z\| \leq \sum_{i=0}^{\nu-k-1} \lambda^{\circ i}\left(\gamma_{w}\right)\right\} \\
& X_{\nu-k} \triangleq X \sim B_{\gamma_{w}}^{\nu-k}=\left\{x \in \mathbb{R}^{n}: x+y \in X, \forall y \in B_{\gamma_{w}}^{\nu-k}\right\}
\end{aligned}
$$

where $\nu \geq k$. In order to introduce the tightened constraints MPC scheme, first let $\boldsymbol{\mu}_{k_{1}, k_{2}} \triangleq$ $\left\{\mu_{k_{1}}, \mu_{k_{1}+1}, \ldots, \mu_{k_{2}}\right\}, k_{2} \geq k_{1}$, be a sequence of control inputs.

Definition 1 ( $F H O C P$ )

Given the positive integer $N$, the stage $\operatorname{cost} l$, the terminal penalty $V_{f}$ and the terminal set $X_{f}$, the Finite Horizon Optimal Control Problem (FHOCP) consists of minimizing, with respect to $\boldsymbol{\mu}_{k, k+N-1}$, the performance index

$$
J\left(\xi, \boldsymbol{\mu}_{k, k+N-1}, N\right) \triangleq \sum_{\nu=k}^{k+N-1} l\left(\xi_{\nu \mid k}, \mu_{\nu}\right)+V_{f}\left(\xi_{k+N \mid k}\right)
$$

subject to

1. the state dynamics (10) with $w_{\nu}=0, \nu=k, \ldots, k+N-1$, and $\xi_{k \mid k}=\xi$;

2. the state constraints $\zeta_{\nu \mid k} \in X_{\nu-k}, \nu=k, \ldots, k+N-1$;

3. the control constraints $\mu_{\nu}^{(j)} \in \underline{U}^{(j)}, j=1, \ldots, m$, and $\nu \in[k, k+N-1]$;

4. the terminal state constraint $\zeta_{k+N \mid k} \in X_{f}$.

According to the receding horizon principle, define $\kappa^{M P C}(\xi)=\mu_{k, k}^{o}(\xi)$ where $\mu_{k, k}^{o}(\xi)$ is the first column of the optimal control sequence $\boldsymbol{\mu}_{k, k+N-1}^{o}$, and apply the control law

$$
\mu=\kappa^{M P C}(\xi)
$$

In the following, $J^{o}(\xi)$ will denote the value of the performance index when the optimal control sequence $\boldsymbol{\mu}_{k, k+N-1}^{o}$ is applied.

\section{Remark 4}

It is of fundamental importance to note that the $F H O C P$ requires the satisfaction of constraint $x \in X$ in (4) only every $M$ steps, i.e. $\xi \in X$, while during the intersampling evolution the state could exceed the constraint. In order to fulfill the constraints even during the intersampling period, the MPC should be designed with respect to system (1), while allowing the change of the MPC input variables only every $M$ time instants; however, this would cause a significative increase on the computational burden. Another possible solution is to impose more conservative state constraints in the FHOCP, in order to be sure that the state value never exceeds $X$ (see, e.g., [22]).

Since the 90s, several ideas have been proposed in the NMPC community in order to provide sufficient conditions on the terminal cost function $V_{f}$ and the terminal constraint $X_{f}$ to guarantee closed-loop stability (see for example [25]). In the following, the least conservative set of necessary assumptions that allow the optimal MPC cost function $J^{o}(\xi)$ to be an ISS-Lyapunov function [21] is reported. Note that, although the FHOCP has been stated for nominal conditions, it is possible to guarantee the regional ISS property [21] of the closed-loop system formed by (10), (22) in a robust positively invariant (RPI) set. The definitions of regional ISS and RPI set and the necessary assumptions are given below.

Definition 2 (RPI set)

A set $\Xi \subset \mathbb{R}^{n}$ is a Robust Positively Invariant (RPI) set for closed-loop system (10), (22), if $\xi_{k+1} \in \Xi, \forall \xi_{k} \in \Xi$ and $\forall w_{k} \in W$. 
Definition 3 (Regional ISS [21])

Given a compact set $\Xi \subset \mathbb{R}^{n}$, if $\Xi$ is RPI for (10), (22), i.e. $\xi_{k+1} \in \Xi, \forall \xi_{k} \in \Xi$ and $\forall w_{k} \in W$, and if there exist a $\mathcal{K} \mathcal{L}$-function $\beta$ and a $\mathcal{K}$-function $\gamma$ such that

$$
|\xi(k, \bar{\xi}, \mathbf{w})| \leq \max \left\{\beta(\|\bar{\xi}\|, k), \gamma\left(\left\|\mathbf{w}_{k-1}\right\|_{\mathcal{L}_{\infty}}\right)\right\},
$$

for all $k \in \mathbb{Z}_{\geq 1}, \forall \bar{\xi} \in \Xi$, then the system (10), (22), with $\mathbf{w} \in \mathcal{M}_{W}$, is said to be Input-to-State Stable $(I S S)$ in $\Xi$.

\section{Assumption 5}

There exist $\mathcal{K}$-functions $\alpha_{l}, \alpha_{1}$ such that

1. $l(x, u) \geq \alpha_{1}(\|\xi\|), \forall \xi \in X, \forall u \in U$;

2. $|l(\xi, u)-l(\zeta, u)| \leq \alpha_{l}(|| \xi-\zeta||), \forall \xi, \zeta \in X, \forall u \in U$.

\section{Assumption 6}

The design parameter $V_{f}$ and the set $\Phi \triangleq\left\{\xi: V_{f}(\xi) \leq \alpha\right\}, \alpha>0$, are such that, given an auxiliary control law $\kappa_{f}$, a $\mathcal{K}$-function $\alpha_{2}$ and $\mathcal{K}_{\infty}$-functions $\alpha_{V_{f}}, \beta_{V_{f}}$

1. $\Phi \subseteq X_{N-1}$

2. $\alpha_{V_{f}}(\|\xi\|) \leq V_{f}(\xi)<\beta_{V_{f}}(\|\xi\|), \forall \xi \in \Phi$

3. $\left|V_{f}(\xi)-V_{f}(\zeta)\right| \leq \alpha_{V}(\|\xi-\zeta\|), \forall \xi, \zeta \in \Phi$;

4. $\kappa_{f}^{(j)}(\xi) \in \underline{U}^{(j)}, \forall j=1, \ldots, m, \forall \xi \in \Phi$

5. $\phi\left(\xi, \kappa_{f}(\xi)\right) \in \Phi, \forall \xi \in \Phi$

6. $V_{f}\left(\phi\left(\xi, \kappa_{f}(\xi)\right)\right)-V_{f}(\xi) \leq-l\left(\xi, \kappa_{f}(\xi)\right), \forall \xi \in \Phi$;

7. $J^{o}(\xi) \leq \alpha_{2}(\xi), \forall \xi \in X^{M P C}(N)$.

\section{Assumption 7}

The design parameter $X_{f} \triangleq\left\{\xi \in \mathbb{R}^{n}: V_{f}(\xi) \leq \alpha_{v}\right\}, \quad \alpha_{v}>0$, is such that, for all $\xi \in \Phi$, $\phi\left(\xi, k_{f}(\xi)\right) \in X_{f}$.

The assumptions above can appear quite difficult to be satisfied, but they are standard in the development of nonlinear stabilizing MPC algorithms, [13,25]. In Section 6 all the assumptions have been verified on a numerical example. In the following we denote $\xi(k, \bar{\xi}, \mathbf{w})$ the solution to the difference equation (10), (22) at time $k$, starting from state $\xi_{0}=\bar{\xi}$ and with inputs $\mathbf{w}$, and $X^{M P C}(N)$ the set of states of the system where an admissible solution of the FHOCP exists.

\section{Theorem 2}

Let a system be described by a model given by (10). Assume that Assumptions 1-7 are satisfied. Then the closed loop system (10), (22) is $I S S$ with RPI set $X^{M P C}(N)$ if the uncertainty is such that

$$
\alpha_{V}\left(\lambda^{N-1}\left(\gamma_{w}\right)\right) \leq \alpha-\alpha_{v}
$$

Proof: The result is immediately obtained from [13].

\subsection{Contribution of MSMDC to MPC performance}

The contribution of the MSMDC to the MPC control strategy can be summarized as follows. As stated in Proposition 3, MSMDC aims at reducing the value $\gamma_{w}$ in condition (24), at expenses of a reduction of the maximum control amplitude available to MPC

$$
\underline{U}^{(j)} \triangleq U^{(j)}-\bar{U}^{(j)}, j=1, \ldots, m,
$$

that, consequently, could lead to a reduction of $X^{M P C}(N)$. In order to mitigate such effect, for a given initial state condition $\xi_{k}$, we could assign to the MSMDC the minimum control amplitude necessary to guarantee feasibility of the FHOCP and ISS. On the other hand, this would clearly increase $\gamma_{w}$, whose value directly affects the size of $D\left(\gamma_{w}\right)$, the set to which the perturbed closedloop system asymptotically converges [21]. A procedure aimed at dynamically modify the MSMDC 
control amplitude, in order to preserve good performance, provide a large domain of attraction, and guarantee $I S S$ with the smallest $D\left(\gamma_{w}\right)$ possible, is therefore needed. A possible idea is the following: among a set of candidates $\bar{U}_{s}^{(j)}$, with $\bar{U}_{s}^{(j)} \leq \bar{U}_{s+1}^{(j)}, j=1, \ldots, m, s=1, \ldots, \bar{s}$, choose, given an initial condition $\xi_{k}$, the one that guarantees feasibility of the FHOCP and ISS with the strongest disturbance reduction. This would require in the worst case the online solution, at each sampling time, of $\bar{s} F H O C P$ problems. In order to alleviate complexity, in Algorithm 1 we propose to solve at each time step two FHOCP only, with $\bar{U}_{s}^{(j)}$ and $\bar{U}_{s+1}^{(j)}$, and select the one with strongest disturbance reduction that provides $I S S$ and feasibility w.r.t. $\xi_{k}$. It is worth noting that the algorithm guarantees recursive feasibility: given $\xi_{k}$, if the $F H O C P$ is feasible at time $k$ and $i$ is kept constant for all $\bar{k} \geq k$, recursive feasibility is guaranteed by standard arguments [13]. On the other hand, the update from $s$ to $s+1$ occurs only if the FHOCP with $\underline{U}^{(j)}=U^{(j)}-\bar{U}_{s+1}^{(j)}$ is feasible.

\section{Remark 5}

Note that allocating as much input as possible to the MSMDC, so as to obtain the strongest reduction of $\gamma_{w}$, results in less tightened state constraints $X_{\nu-k}$ (see Definition 1), and allows to increase $N$ while still preserving $I S S$ (see eq. (24)). The combination of these effects could lead to an enlargement of $X^{M P C}(N)$ but, on the other hand, this will be achieved at the cost of less input available to the MPC, and, therefore, at the cost of a possible degradation of the control performance.

In conclusion, we summarize the advantages and the disadvantages of the proposed scheme with respect to the NMPC and the SM control strategies used separately:

- Advantages w.r.t robust NMPC only: robust NMPC aims to guarantee theoretical properties (ISS of the closed-loop system, constraint satisfaction) in the presence of disturbances. If the disturbances are too large, the MPC controller by itself can be not feasible. The effect of MSMDC is of reducing the equivalent disturbance visible by the NMPC scheme, thus possibly recovering feasibility.

- Disadvantages w.r.t MPC only: In absence of a dynamic input allocation scheme, it could lead to a reduction of the feasibility domain. Moreover, it is necessary to set up a multirate control strategy, which requires a slightly larger computational effort.

- Advantages w.r.t sliding mode control only: constraints satisfaction and optimal control. The nominal performance are affected by the choice of the cost function parameters.

- Disadvantages w.r.t sliding mode control only: heavier computational burden, since the NMPC controller is computationally much more demanding than any sliding mode controller.

\section{NUMERICAL EXAMPLE}

The control strategy proposed in this paper has been applied in simulation to the two-dimensional system

$$
x_{t+1}=\left[\begin{array}{cc}
\left(1-0.6 T_{s}\right) & -T_{s} \\
T_{s} & 1
\end{array}\right] x_{t}+\left[\begin{array}{c}
T_{s} \\
0
\end{array}\right] u_{t}+\left[\begin{array}{cc}
0 & -T_{s} \\
0 & 0
\end{array}\right] x_{t}^{3}+\left[\begin{array}{c}
T_{s} \\
0
\end{array}\right] d_{t},
$$

with $T_{s}=0.001$, which was already used in [5] to test another NMPC algorithm. The input and state constraints are $U=\{u \in \mathbb{R}:|u| \leq 2.5\}$ and $X=\left\{x \in \mathbb{R}^{2}:\|x\|_{\infty} \leq 1.3\right\}$. We assume that $|d| \leq$ 0.25 , and $\left|d_{t+1}-d_{t}\right| \leq 0.15$ and, therefore, $\delta=\left[2.5 \cdot 10^{-4} 0\right]^{\top}$ and $\gamma_{\delta}=2.5 \cdot 10^{-4}$. The functions in Assumption 2 have been chosen as $\eta_{(j)}(\|a-b\|)=\bar{\eta}_{(j)} \cdot\|a-b\|$, and $\eta(\|a-b\|)=\bar{\eta} \cdot\|a-b\|$, with $\bar{\eta}_{(1)}=0.9994, \bar{\eta}_{(2)}=1$, and $\bar{\eta}=1.0026$. The values of $\bar{\eta}_{(j)}$ and $\bar{\eta}$ have been computed using the INTLAB interval toolbox [36]. For the HLC, we choose $M=100$. The bounds on the disturbance terms for the M-sampled system without MSMDC, $\omega=\left[\begin{array}{ll}0.0284 & 0.0281\end{array}\right]^{\top}, \gamma_{w}=0.0285$, have been computed according to Lemma 1 while $\lambda(\|a-b\|)=\bar{\lambda} \cdot\|a-b\|$, with $\bar{\lambda}=1.2929$, has been computed according to Lemma 2. As performance index of the FHOCP we choose a quadratic 


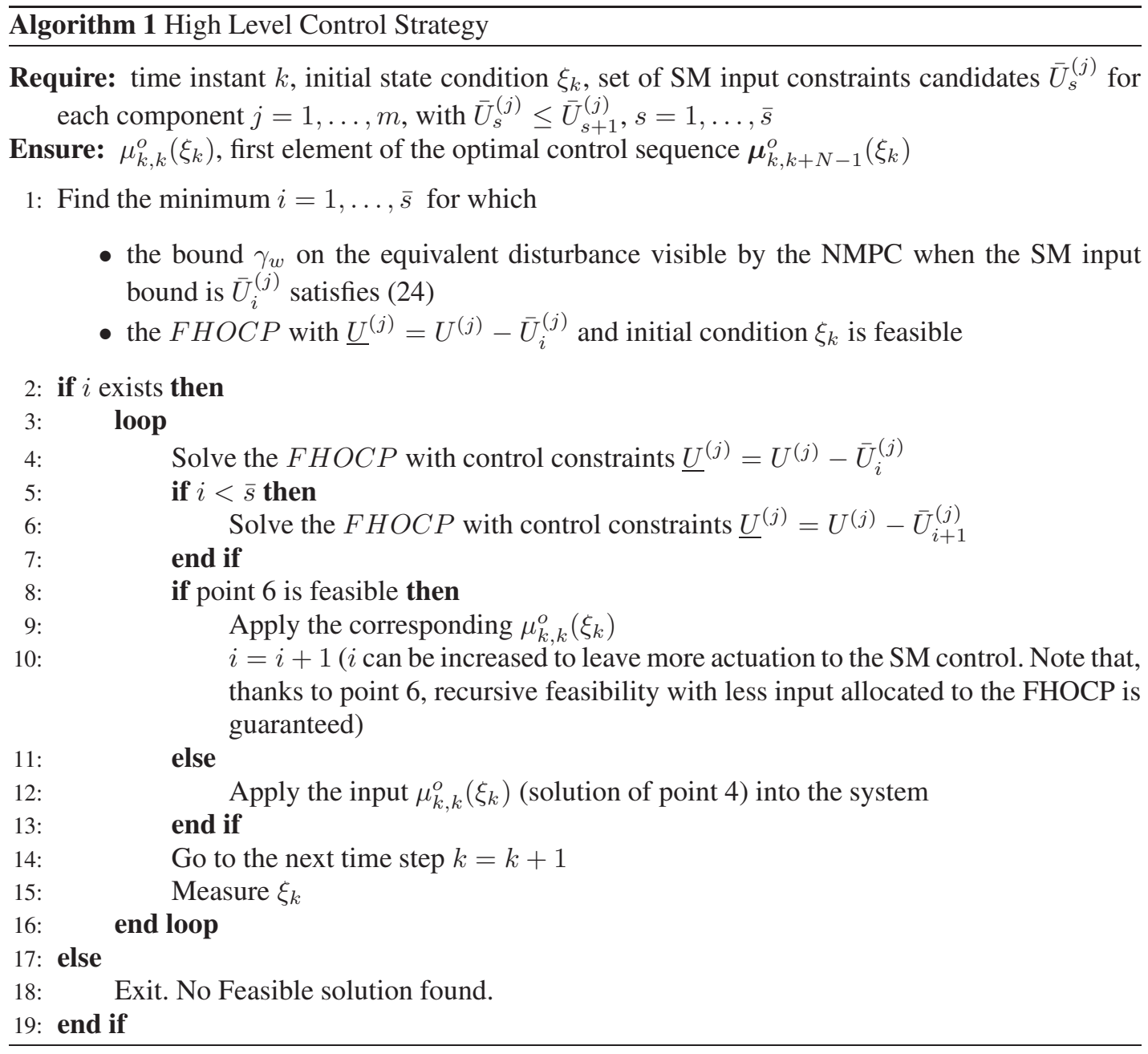

cost function

$$
J\left(\bar{\xi}, \mu_{k, k+N-1}, N\right) \triangleq \sum_{\nu=k}^{k+N-1}\left(\zeta_{\nu \mid k}^{\top} Q \zeta_{\nu \mid k}+\mu_{\nu}^{\top} R \mu_{\nu}\right)+\zeta_{k+N \mid k}^{\top} \Pi \zeta_{k+N \mid k}
$$

with prediction horizon $N=7$, and parameters

$$
Q=\left[\begin{array}{ll}
1 & 0 \\
0 & 1
\end{array}\right], R=0.01, \Pi=\left[\begin{array}{cc}
8.0660 & 3.4017 \\
3.4017 & 20.6744
\end{array}\right]
$$

The matrix $\Pi$ is related to the unconstrained infinite horizon solution $P$ of the discrete-time Riccati equation, and has been computed according to [18]. The functions defined in Assumptions 5 have been determined as $\alpha_{1}(\|\xi\|)=\|\xi\|^{2}, \alpha_{l}(\|\xi-\zeta\|)=3.6770 \cdot\|\xi-\zeta\|$. The auxiliary control law $\kappa_{f}$ in Assumption 6 has been chosen as $\kappa_{f} \triangleq K_{f} \xi$ where $K_{f}=\left[\begin{array}{ll}-1.9563 & -0.8825\end{array}\right]$ is the state feedback gain of the optimal LQR controller. The other functions and parameters in Assumption 6 are $V_{f}(\xi)=\xi^{\top} \Pi \xi, \alpha=0.5074, \alpha_{V_{f}}(\|\xi-\zeta\|)=7.2068 \cdot\|\xi-\zeta\|^{2}, \beta_{V_{f}}(\|\xi-\zeta\|)=21.5336$. $\|\xi-\zeta\|^{2}, \alpha_{V}(\|\xi-\zeta\|)=3.7553 \cdot\|\xi-\zeta\|$ (this latter has been computed using the INTLAB interval toolbox), while a value for $\alpha_{2}$ can be obtained from $\alpha_{V}(\cdot)$, as mentioned in [13] and [15]. A value for the parameter $\alpha_{v}$ in Assumption 7 has been obtained using the INTLAB interval toolbox, as $\alpha_{v}=0.1617$. 
Table I. Bounds on the HLC disturbance term for different values of $\bar{U}_{R e q(i)}$

\begin{tabular}{|c|c|c|c|c|}
\hline$s$ & $i$ & $\bar{U}_{\operatorname{Req}(i)}^{(1)}$ & $\omega$ & $\gamma_{w}$ \\
\hline 1 & 0 & 0.2500 & {$\left[\begin{array}{lll}0.0150 & 0.0149\end{array}\right]^{\top}$} & 0.0150 \\
\hline 2 & 19 & 0.9035 & {$\left[\begin{array}{lll}0.0112 & 0.0117\end{array}\right]^{\top}$} & 0.0119 \\
\hline 3 & 39 & 1.1862 & {$\left[\begin{array}{lll}0.0078 & 0.0086\end{array}\right]^{\top}$} & 0.0087 \\
\hline 4 & 59 & 1.4015 & {$\left[\begin{array}{lll}0.0044 & 0.0054\end{array}\right]^{\top}$} & 0.0056 \\
\hline 5 & 79 & 1.5825 & {$\left[\begin{array}{lll}0.0011 & 0.0023\end{array}\right]^{\top}$} & 0.0025 \\
\hline 6 & 99 & 1.7416 & {$\left[\begin{array}{lll}0.0001 & 0.0015\end{array}\right]^{\top}$} & 0.0015 \\
\hline
\end{tabular}

According to Theorem 2, ISS is guaranteed if condition (24) is satisfied. When the tightened NMPC method proposed in [13] is used alone, this condition does not hold since

$$
\bar{\alpha}_{V} \cdot \bar{\lambda}^{N-1} \cdot \gamma_{w}=3.7553 \cdot 1.2929^{6} \cdot 0.0285=0.4992>\alpha-\alpha_{v}=0.3458 .
$$

On the other hand, if the robust open-loop MPC is supported by the sliding mode disturbance compensator with $\bar{U}^{(1)}=\bar{U}_{\operatorname{Req}(M-1)}^{(1)}=1.7416$, the disturbance term seen by the HLC is $\omega=$ $\left[\begin{array}{ll}1.5 \cdot 10^{-4} & 0.0015\end{array}\right]^{\top}, \gamma_{w}=0.0015$. In this case, condition (24) is satisfied,

$$
\bar{\alpha}_{V} \cdot \bar{\lambda}^{N-1} \cdot \gamma_{w}=0.0263<\alpha-\alpha_{v}=0.3458,
$$

but the input amplitude left to MPC is $\underline{U}^{(1)}=\{u \in \mathbb{R}:|u| \leq 0.7584\}$ only. Due to the tighter input constraints, the cost to pay for $I S S$ is a reduction of $X^{M P C}(N)$. To mitigate such effect, we make use of the results in Theorem 1 and Algorithm 1. A sketch of the resulting domain of attraction $X^{M P C}(7)$ is reported in Figure 2. Table I reports the upper bound on the HLC disturbance term for some of the values of $\bar{U}_{\operatorname{Req}(i)}^{(1)}, i=0, \ldots, 99$. ISS is guaranteed for all the cases reported in the table. In the following, Algorithm 1 has been implemented using as candidates $\bar{U}_{s}^{(1)}$ only the values of $\bar{U}_{R e q(i)}^{(1)}$ reported in the table. Given the initial condition $x_{t}=\left[\begin{array}{lll}-1.2188 & 0.4063\end{array}\right]^{\top}$, the maximum value of $\bar{U}^{(1)}$ that still allows to maximally counteract the disturbances while guaranteeing feasibility of the FHOCP and $I S S$ stability was $\bar{U}_{\text {Req(19) }}^{(1)}=0.9035$. Figure 3 shows the evolution of the states (left) and the inputs (right) when the strategy in Algorithm 1 is applied. The multirate sliding mode disturbance compensation acts at a faster rate and compensate the disturbance in Figure 4 (right) in a very effective way (note that the actual realization of $d_{t}$ is a rapidly-varying signal with zero mean). According to the algorithm, the bounds of the HLC and the MSMDC are varying in time (see Figure 4 (left)). While at the beginning $\bar{U}_{\operatorname{Req}(19)}^{(1)}=0.9035$ is required, after few steps the strategy switches to $\bar{U}_{R e q(39)}^{(1)}$, then to $\bar{U}_{R e q(59)}^{(1)}$ for two steps, then to $\bar{U}_{R e q(79)}^{(1)}$ for other two steps, and finally, when the state gets close to the origin and less MPC control amplitude is required in order to provide feasibility of the FHOCP, to $\bar{U}_{\operatorname{Req}(99)}^{(1)}=1.7416$, thus providing to MSMDC the maximum control amplitude required for the disturbance rejection.

Finally, Figure 5 shows the advantages of the proposed solution w.r.t. the direct discretization of a continuous-time SM controller, as the one proposed in [33]. The direct application of the discontinuous control law in this case would lead to

$$
\bar{u}_{t}^{(j)}=-\bar{U}_{s}^{(j)} \operatorname{sign}\left(s_{t}^{(j)}\right)
$$

where $s_{t}^{(j)}$ is the component of $s_{t}^{(j)}$ relative to the $j-t h$ state variable. One can notice that the proposed approach leads to a reduction of the chattering effect of about one order of magnitude w.r.t. the directly-discretized scheme. The advantage of the MSMDC will be more apparent as the value of $T_{s}$ increases. 


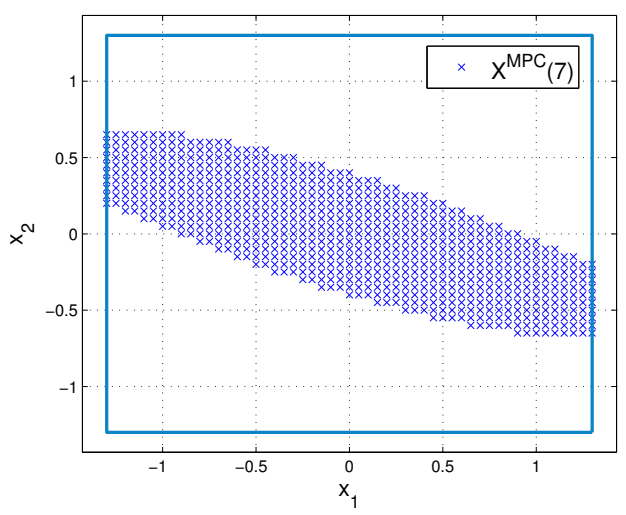

Figure 2. Domain of attraction when Algorithm 1 is applied.
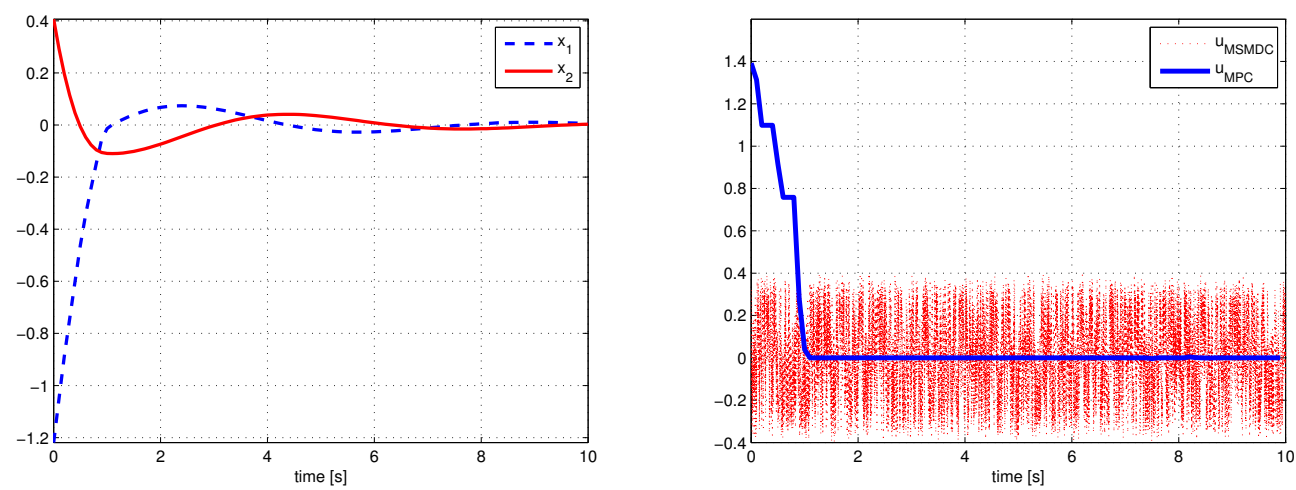

Figure 3. State evolution (left) and input sequence (right).
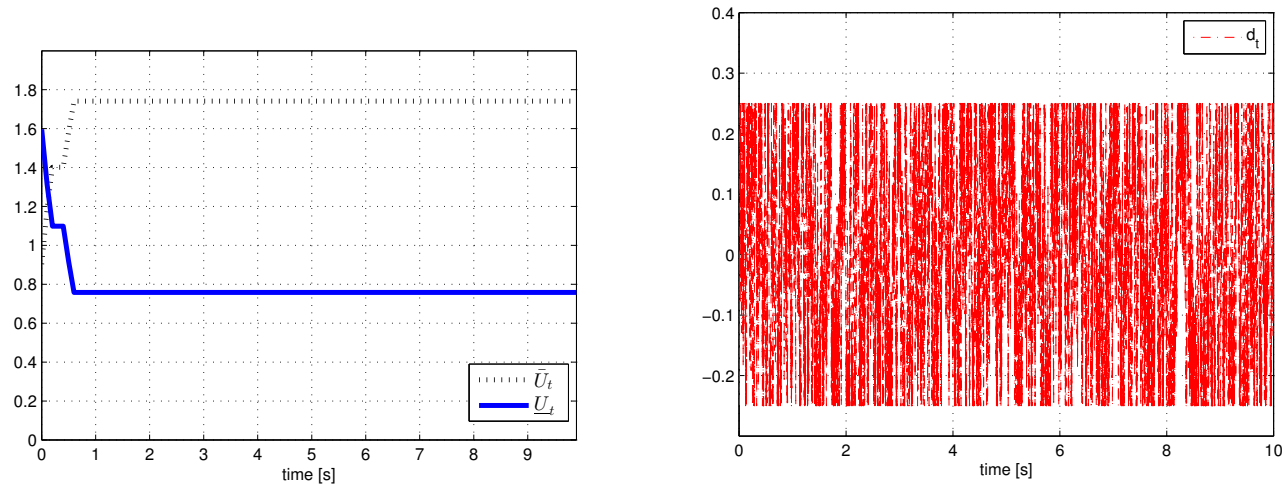

Figure 4. Input bounds (left) and noise sequence (right).

\section{CONCLUSIONS}

The paper introduced a multirate control strategy for nonlinear systems which combines robust MPC and discrete-time sliding mode control. It is shown how the use of the proposed strategy can reduce the conservativeness and enhance the performance of the MPC controller, in terms of region of 


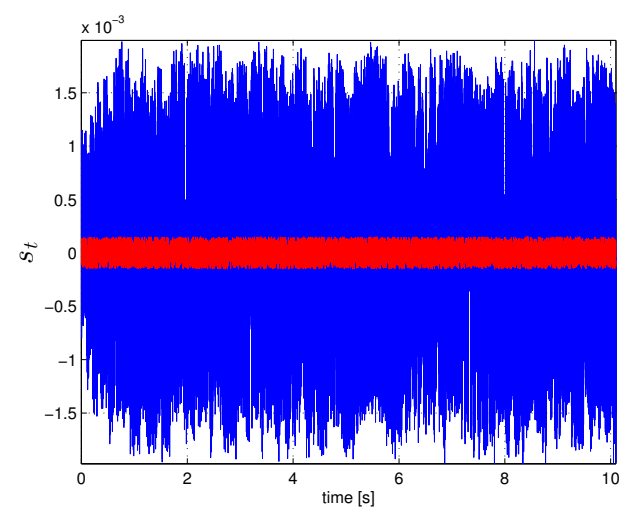

Figure 5. Comparison of the sliding variable obtained applying the MSMDC strategy (red) and the one in equation (27) (blue).

attraction and set where the state of the closed-loop system ultimately converges. The algorithm has been tested on a simple simulation example, which shows the advantages of the proposed technique.

\section{APPENDIX}

Proof of Lemma 1: Given equations (1) and (8), it is possible to see that $\left|x_{t M}^{(j)}-z_{t M \mid t M}^{(j)}\right|=0$ and

$$
\begin{aligned}
\left|x_{t M+1}^{(j)}-z_{t M+1 \mid t M}^{(j)}\right| & =\left|f^{(j)}\left(x_{t M}\right)+d_{t M}^{(j)}-f^{(j)}\left(z_{t M \mid t M}\right)\right| \\
& \leq\left|f^{(j)}\left(x_{t M}\right)-f^{(j)}\left(z_{t M \mid t M}\right)\right|+\left|d_{t M}^{(j)}\right| \\
& \leq \eta_{(j)}\left(\varrho_{0}\right)+\delta^{(j)}=\sigma_{1}^{(j)}
\end{aligned}
$$

where the last step is obtained by applying the definition of $\sigma_{i}^{(j)}$ and noting that $\varrho_{0}=$ $\min \left(\left\|\sigma_{0}\right\|, \zeta_{0}\right)=0$ and $\eta_{(j)}(0)=0$, being $\eta_{(j)}(\cdot)$ a $\mathcal{K}$-function. It results that

$$
\begin{aligned}
\left\|x_{t M+1}-z_{t M+1 \mid t M}\right\| & \leq \gamma_{\delta} \\
& =\min \left(\left\|\sigma_{1}\right\|, \zeta_{1}\right)=\varrho_{1}
\end{aligned}
$$

where the last step comes from the definition of $\sigma_{1}, \zeta_{1}$ noting that $\eta(0)=0$, being $\eta(\cdot)$ a $\mathcal{K}$-function. If we keep iterating, using Assumption 2, one has

$$
\begin{aligned}
\left|x_{t M+2}^{(j)}-z_{t M+2 \mid t M}^{(j)}\right| & \leq \eta_{(j)}\left(\| x_{t M+1}-z_{t M+1 \mid t M}||\right)+\delta^{(j)} \\
& \leq \eta_{(j)}\left(\varrho_{1}\right)+\delta^{(j)}=\sigma_{2}^{(j)}
\end{aligned}
$$

and from this it follows that

$$
\begin{aligned}
\left\|x_{t M+2}-z_{t M+2 \mid t M}\right\| & =\left\|f\left(x_{t M+1}\right)+d_{t M+1}-f\left(z_{t M+1 \mid t M}\right)\right\| \\
& \leq\left\|f\left(x_{t M+1}\right)-f\left(z_{t M+1 \mid t M}\right)\right\|+\left\|d_{t M+1}\right\| \\
& \leq \eta\left(\left\|x_{t M+1}-z_{t M+1 \mid t M}\right\|\right)+\gamma_{\delta} \\
& \leq \eta\left(\varrho_{1}\right)+\gamma_{\delta}=\zeta_{1}
\end{aligned}
$$

In order to get the tightest upper bound possible for $\left\|x_{t M+2}-z_{t M+2 \mid t M}\right\|$ we make use of (28) and (29)

$$
\left\|x_{t M+2}-z_{t M+2 \mid t M}\right\| \leq \min \left(\left\|\sigma_{2}\right\|, \zeta_{2}\right)=\varrho_{2}
$$


Iterating this procedure, after $M$ sampling times one obtains (12).

Proof of Lemma 3: note first that (16) implies $\left|x_{t M+i}^{(j)}-z_{t M+i \mid t M}^{(j)}\right|=\left|d_{i-1}^{(j)}-\hat{d}_{i-1}^{(j)}\right|$ for $i=1, . ., M$ and $j=1, . ., m$, which leads to $\left|x_{t M+i}^{(j)}-z_{t M+i \mid t M}^{(j)}\right|=e^{(j)}, j=1, . ., m$. For the same reasons as in Lemma 1, it is possible to see that $\left|x_{t M+1}^{(j)}-z_{t M+1 \mid t M}^{(j)}\right| \leq \eta_{(j)}\left(\hat{\varrho}_{0}\right)+\delta^{(j)}=\hat{\sigma}_{1}^{(j)}, \forall j=m+1, . ., n$ and $\left\|x_{t M+1}-z_{t M+1 \mid t M}\right\| \leq \gamma_{e}=\min \left(\left\|\hat{\sigma}_{1}\right\|, \hat{\zeta}_{1}\right)=\hat{\varrho}_{1}$. If we keep iterating, using Assumption 2, one has

$$
\begin{aligned}
\left|x_{t M+2}^{(j)}-z_{t M+2 \mid t M}^{(j)}\right| & \leq \eta_{(j)}\left(\left\|x_{t M+1}-z_{t M+1 \mid t M}\right\|\right)+\delta^{(j)} \\
& \leq \eta_{(j)}\left(\hat{\varrho}_{1}\right)+\delta^{(j)}=\hat{\sigma}_{2}^{(j)}
\end{aligned}
$$

for all $j=m+1, . ., n$, and from this comes also the fact that

$$
\begin{aligned}
\left\|x_{t M+2}-z_{t M+2 \mid t M}\right\| & \leq \eta^{[m+1, n]}\left(\left\|x_{t M+1}-z_{t M+1 \mid t M}\right\|\right)+\gamma_{e} \\
& \leq \eta^{[m+1, n]}\left(\hat{\varrho}_{1}\right)+\gamma_{e}=\hat{\zeta}_{2}
\end{aligned}
$$

In order to get the tightest upper bound possible for $\left\|x_{t M+2}-z_{t M+2 \mid t M}\right\|$ we make use of (30) and (31)

$$
\left\|x_{t M+2}-z_{t M+2 \mid t M}\right\| \leq \min \left(\left\|\hat{\sigma}_{2}\right\|, \hat{\zeta}_{2}\right)=\hat{\varrho}_{2}
$$

Iterating this procedure, after $M$ sampling times one obtains (17).

The maximum amplitude of the MSMDC control variable needed up to time instant $t M+i$ to compensate for the matched disturbance can be determined as follows. By definition, each component of MSMDC control law $\bar{u}_{t M+i}(i=0, \cdots, M-1)$ can be written as

$$
\bar{u}_{t M+i}^{(j)}=-f^{(j)}\left(x_{t M+i}\right)+f^{(j)}\left(z_{t M+i \mid t M}\right)-\hat{d}_{t M+i}^{(j)}
$$

Then, using Assumption 2 and equation (32), one has

$$
\left|\bar{u}_{t M}^{(j)}\right| \leq \delta^{(j)}, \quad\left|\bar{u}_{t M+1}^{(j)}\right| \leq \eta_{(j)}\left(\varrho_{1}\right)+\delta^{(j)}
$$

Iterating this procedure until $i=M-1$, one obtains the bound in (19).

Proof of Theorem 1: First, note that the bound (19) is easily obtainable from the proof of Lemma 3 , assuming that such an input is available to the MSMDC controller. Indeed this holds for all $i \leq \underline{i}$. Also, note that $\breve{\sigma}_{i}^{(j)}=\hat{\sigma}_{i}^{(j)}, \breve{\zeta}_{i}=\hat{\zeta}_{i}, \breve{\varrho}_{i}=\hat{\varrho}_{i}$, for $i \leq \underline{i}$, since there is enough control input to compensate for the disturbance for all components $j \in\{1, \ldots, m\}$.

If $i=\underline{i}+1$, then

$$
\begin{aligned}
\left|x_{t M+\underline{i}+1}^{(j)}-z_{t M+\underline{i}+1 \mid t M}^{(j)}\right|= & \left|f^{(j)}\left(x_{t M+\underline{i}}\right)+\underline{u}_{t M+\underline{i}}^{(j)}+\bar{u}_{t M+\underline{i}}^{(j)}+d_{t M+\underline{i}-1}^{(j)}+-f^{(j)}\left(z_{t M \mid t M}\right)-\underline{u}_{t M+\underline{i}}^{(j)}\right| \\
= & \left|f^{(j)}\left(x_{t M+\underline{i}}\right)+\bar{u}_{t M+\underline{i}}^{(j)}+d_{t M+\underline{i}}^{(j)}-f^{(j)}\left(z_{t M+\underline{i} \mid t M}\right)\right| \\
= & \mid f^{(j)}\left(x_{t M+\underline{i}}\right)-\operatorname{sat}\left(f^{(j)}\left(x_{t M+\underline{i}}\right)-f^{(j)}\left(z_{t M+\underline{i} \mid t M}\right)+\hat{d}_{t M+(\underline{i)}}^{(j)}\right)+ \\
& +d_{t M+\underline{i}}^{(j)}-f^{(j)}\left(z_{t M+\underline{i} \mid t M) \mid}\right) \\
= & \mid f^{(j)}\left(x_{t M+\underline{i}}\right)-\operatorname{sat}\left(f^{(j)}\left(x_{t M+\underline{i}}\right)-f^{(j)}\left(z_{t M+\underline{i} \mid t M}\right)+\hat{d}_{t M+(\underline{i)})}^{(j)}\right)+ \\
& +\hat{d}_{t M+\underline{i}}^{(j)}-\hat{d}_{t M+\underline{i}}^{(j)}+d_{t M+\underline{i}}^{(j)}-f^{(j)}\left(z_{t M+\underline{i} \mid t M}\right) \mid \\
\leq & \mid f^{(j)}\left(x_{t M+\underline{i}}\right)-f^{(j)}\left(z_{t M+\underline{i} \mid t M}\right)+\hat{d}_{t M+\underline{i}}^{(j)}-\operatorname{sat}\left(f^{(j)}\left(x_{t M+\underline{i}}\right)+\right. \\
& \left.-f^{(j)}\left(z_{t M+\underline{i} \mid t M}\right)+\hat{d}_{t M+\underline{i}}^{(j)}\right) \mid+e^{(j)} \\
\leq & \min \left(\eta_{(j)}\left(\hat{\varrho}_{\underline{i}}\right)+\delta^{(j)}, \max \left(e^{(j)}, \eta_{(j)}\left(\hat{\varrho}_{\underline{i}}\right)+\delta^{(j)}-\bar{U}^{(j)}+e^{(j)}\right)\right) \\
= & \min \left(\eta_{(j)}\left(\breve{\varrho}_{\underline{i}}\right)+\delta^{(j)}, \gamma_{\underline{i}+1}^{(j)}\right) \\
= & \breve{\sigma}_{\underline{i}+1}^{(j)}
\end{aligned}
$$


for $j \in\{1, \ldots, m\}$, where

$$
\operatorname{sat}\left(\hat{d}_{t M}^{(j)}\right)= \begin{cases}\hat{d}_{t M}^{(j)} & \text { if }\left|\hat{d}_{t M}^{(j)}\right| \leq \bar{U}^{(j)} \\ \operatorname{sign}\left(\hat{d}_{t M}^{(j)}\right) \bar{U}^{(j)} & \text { if }\left|\hat{d}_{t M}^{(j)}\right|>\bar{U}^{(j)}\end{cases}
$$

The max indicates that the minimum bound is the one obtained in Lemma 3, while the min indicates that the maximum bound is the one obtained in Lemma 1. An upper bound for $\| x_{t M+\underline{i}+1}-$ $z_{t M+\underline{i}+1 \mid t M} \|$ is given by $\left\|\breve{\sigma}_{\underline{i}}\right\|$. We also know that

$$
\begin{aligned}
\left|x_{t M+\underline{i}+1}^{(j)}-z_{t M+\underline{i}+1 \mid t M}^{(j)}\right| \leq & \mid f^{(j)}\left(x_{t M+\underline{i}}\right)-f^{(j)}\left(z_{t M+\underline{i} \mid t M}\right)+\hat{d}_{t M+\underline{\underline{i}}}^{(j)}-\operatorname{sat}\left(f^{(j)}\left(x_{t M+\underline{i}}\right)+\right. \\
& \left.-f^{(j)}\left(z_{t M+\underline{i} \mid t M}\right)+\hat{d}_{t M+\underline{(i)}}^{(j)}\right)+\hat{d}_{t M+\underline{(i)}}^{(j)}-\hat{d}_{t M+\underline{(i)}}^{(j)} \mid \\
\leq & \left|f^{(j)}\left(x_{t M+\underline{i}}\right)-f^{(j)}\left(z_{t M+\underline{i} \mid t M}\right)\right|+\min \left(\delta^{(j)}, \max \left(e^{(j)}, \delta^{(j)}+\right.\right. \\
& \left.-\bar{U}^{(j)}+e^{(j)}\right) \\
= & \left|f^{(j)}\left(x_{t M+\underline{i}}\right)-f^{(j)}\left(z_{t M+\underline{i} \mid t M}\right)\right|+\delta_{r e d}^{(j)}
\end{aligned}
$$

This bound on $\left|x_{t M+\underline{i}+1}^{(j)}-z_{t M+i+1 \mid t M}^{(j)}\right|$ is more conservative than the previous one but helps in the construction of a tighter bound for $\left\|x_{t M+\underline{i}+1}-z_{t M+\underline{i}+1 \mid t M}\right\|$. In fact,

$$
\left\|x_{t M+\underline{i}+1}-z_{t M+\underline{i}+1 \mid t M}\right\| \leq \eta\left(\breve{\varrho}_{\underline{i}}\right)+\gamma_{0}=\breve{\zeta}_{\underline{i}+1}
$$

and, in conclusion,

$$
\begin{aligned}
\left\|x_{t M+\underline{i}+1}-z_{t M+\underline{i}+1 \mid t M}\right\| & \leq \min \left(\left\|\breve{\sigma}_{\underline{i}+1}\right\|, \breve{\zeta}_{\underline{i}+1}\right) \\
& =\breve{\varrho}_{\underline{i}+1}
\end{aligned}
$$

Following the proof of Lemma 3 , one gets the bounds on the other components $j \in\{m+1, \ldots, n\}$. Finally, keeping iterating as in the proof of Lemma 3, one gets the bound in equation (20).

\section{REFERENCES}

1. K. Abidi, J. X. Xu, and Y. Xinghuo. On the discrete-time integral sliding-mode control. IEEE Transactions on Automatic Control, 52(4):709-715, 2007.

2. G. Bartolini, A. Ferrara, and V. I. Utkin. Adaptive sliding mode control in discrete-time systems. Automatica, 31(5):769-773, 1995.

3. G. Bartolini, S. Sanna, and E. Usai. Robust near-optimal control via unchattering sliding mode control. In AIP Conference Proceedings, volume 437, page 269, 1998.

4. J. Björnberg and M. Diehl. Approximate robust dynamic programming and robustly stable mpc. Automatica, 42(5):777-782, 2006.

5. M. Canale, L. Fagiano, and M. Milanese. Efficient model predictive control for nonlinear systems via function approximation techniques. IEEE Transactions on Automatic Control, 55(8):1911-1916, 2010.

6. P. Colaneri, R. Scattolini, and N. Schiavoni. Stabilization of multirate sampled-data linear systems. Automatica, 26(2):377-380, 1990.

7. C. Edwards and S. Spurgeon. Sliding mode control: theory and applications. Taylor \& Francis, 1998.

8. W. Garcia-Gabin and E. F. Camacho. Sliding mode model based predictive control for non minimum phase systems. In Proc. Eur. Contr. Conf., Cambridge, UK, September 2003.

9. G. Grimm, M.J. Messina, S.E. Tuna, and A.R. Teel. Nominally robust model predictive control with state constraints. IEEE Transactions on Automatic Control, 52(10):1856-1870, 2007.

10. L. Grüne and J. Pannek. Nonlinear Model Predictive Control: Theory and Algorithms. Springer Verlag, 2011.

11. S Janardhanan and Neeli Satyanarayana. Multirate functional observer based discrete-time sliding mode control. In Advances in Sliding Mode Control, pages 267-281. Springer, 2013.

12. Kyung-Soo Kim and Keun-Ho Rew. Discrete-time disturbance observers with multirate output state estimation. In Proceedings of the International Conference on Control, Automation and Systems, pages 103-106, Gyeonggi-do, Republic of Korea, October 2011.

13. M. Lazar and W. P. M. H. Heemels. Predictive control of hybrid systems: Input-to-state stability results for suboptimal solutions. Automatica, 45(1):180-185, 2009.

14. M. Lazar, D. Munoz de la Pena, W. Heemels, and T. Alamo. On input-to-state stability of min-max nonlinear model predictive control. Systems \& Control Letters, 57(1):39-48, 2008. 
15. D. Limon, T. Alamo, and E. F. Camacho. Input-to-state stable MPC for constrained discrete-time nonlinear systems with bounded additive uncertainties. In Proceedings of the Conference on Decision and Control, Las Vegas, NV, December 2002.

16. D. Limon, T. Alamo, D. M. Raimondo, D. Muñoz de la Peña, J. M. Bravo, A. Ferramosca, and E. F. Camacho. Input-to-state stability: a unifying framework for robust model predictive control. In L. Magni, D. M. Raimondo, and F. Allgöwer, editors, Nonlinear Model Predictive Control: Towards New Challenging Applications, pages 1-26. Springer-Verlag, 2009.

17. D. Limon, T. Alamo, F. Salas, and EF Camacho. Input to state stability of min-max mpc controllers for nonlinear systems with bounded uncertainties. Automatica, 42(5):797-803, 2006.

18. L. Magni, G. De Nicolao, L. Magnani, and R. Scattolini. A stabilizing model-based predictive control algorithm for nonlinear systems. Automatica, 37(9):1351-1362, 2001.

19. L Magni, G De Nicolao, R Scattolini, and F Allgöwer. Robust model predictive control for nonlinear discrete-time systems. International Journal of Robust and Nonlinear Control, 13(3-4):229-246, 2003.

20. L. Magni, D. M. Raimondo, and F. Allgöwer, editors. Nonlinear Model Predictive Control: Towards New Challenging Applications. Springer-Verlag, 2009.

21. L Magni, DM Raimondo, and R Scattolini. Regional input-to-state stability for nonlinear model predictive control. IEEE Transactions on Automatic Control, 51(9):1548-1553, 2006.

22. L. Magni and R. Scattolini. Model predictive control of continuous-time nonlinear systems with piecewise constant control. IEEE Transactions on Automatic Control, 49(6):900-906, 2004.

23. L. Magni and R. Sepulchre. Stability margins of nonlinear receding-horizon control via inverse optimality. Systems \& Control Letters, 32(4):241-245, 1997.

24. D. Q. Mayne, E. C. Kerrigan, E. J. van Wyk, and P. Falugi. Tube-based robust nonlinear model predictive control. International Journal of Robust and Nonlinear Control, 21(11):1341-1353, 2011.

25. David Q Mayne, James B Rawlings, Christopher V Rao, and Pierre OM Scokaert. Constrained model predictive control: Stability and optimality. Automatica, 36(6):789-814, 2000.

26. C. Milosavljevic. General conditions for the existence of a quasi-sliding mode on the switching hyperplane in discrete variable structure systems. Automation and Remote Control, 46:307-314, 1985.

27. G. Pannocchia, J. B. Rawlings, and S. J. Wright. Conditions under which suboptimal nonlinear MPC is inherently robust. Systems \& Control Letters, 60(9):747 - 755, 2011.

28. B. Picasso, D. Desiderio, and R. Scattolini. Robust stability analysis of nonlinear discrete-time systems with application to MPC. IEEE Transactions on Automatic Control, PP(99):1, 2011.

29. G. Pin, D.M. Raimondo, L. Magni, and T. Parisini. Robust model predictive control of nonlinear systems with bounded and state-dependent uncertainties. IEEE Transactions on Automatic Control, 54(7):1681-1687, 2009.

30. K. R. Muske, H. Ashrafiuon, and M. Nikkhah. A predictive and sliding mode cascade controller. In Proceedings of the American Control Conference, New York City, NY, July 2007.

31. D. M. Raimondo, D. Limon, M. Lazar, L. Magni, and E. F. Camacho. Min-max model predictive control of nonlinear systems: A unifying overview on stability. European Journal of Control, 15(1):5-21, 2009.

32. J.B. Rawlings and D.Q. Mayne. Model predictive control: Theory and design. Nob Hill Pub., 2009.

33. M. Rubagotti, A. Estrada, F. Castaños, A. Ferrara, and L. Fridman. Integral sliding mode control for nonlinear systems with matched and unmatched perturbations. IEEE Transactions on Automatic Control, 56(11):2699-2704, 2011.

34. M. Rubagotti, D. M. Raimondo, A. Ferrara, and L. Magni. Robust Model Predictive Control with Integral Sliding Mode in Continuous-Time Sampled-Data Nonlinear Systems. IEEE Transactions on Automatic Control, 56(3):556-570, 2011.

35. M. Rubagotti, D. M. Raimondo, C. N. Jones, L. Magni, A. Ferrara, and M. Morari. A nonlinear model predictive control scheme with multirate integral sliding mode. In Proc. of the IFAC Symposium on Nonlinear Control Systems (NOLCOS), Bologna, Italy, September 2010.

36. S.M. Rump. INTLAB - INTerval LABoratory. In Tibor Csendes, editor, Developments in Reliable Computing, pages 77-104. Kluwer Academic Publishers, Dordrecht, 1999. http://www.ti3.tu-harburg.de/rump/.

37. S. Sarpturk, Y. Istefanopulos, and O. Kaynak. On the stability of discrete-time sliding mode control systems. IEEE Transactions on Automatic Control, 32(10):930-932, 1987.

38. P.O.M. Scokaert and D.Q. Mayne. Min-max feedback model predictive control for constrained linear systems. IEEE Transactions on Automatic Control, 43(8):1136-1142, 1998

39. V. I. Utkin. Sliding mode in control and optimization. Springer-Verlag, 1992.

40. V.I. Utkin, J. Guldner, and J. Shi. Sliding mode control in electromechanical systems. Taylor \& Francis, 1999.

41. Qingsong Xu and Yangmin Li. Model predictive discrete-time sliding mode control of a nanopositioning piezostage without modeling hysteresis. IEEE Transactions on Control Systems Technology, 20(4):983-994, 2012. 